3 庞素珍, 秦静芬, 乐加昌. 竹红菌乙素溴代物对 Hela 细胞形态结构的光敏损伤. 生物物理学报, 1994, 10( 4)：651 656

4 许以明, 张志义, 徐国瑞. 治癌药物 YHPD 对 DNA 光敏损伤的激光喇曼光谱研究. 科学通报. 1989, (19)：1 500 1504

5 赵开弘, 蒋丽金. 竹红菌甲素在碱性和中性介质中的转化. 有机化学, 1989, 9: 252 254

6 George J, Thomas J, Yoshimasa Kyogoku. Biological Science. In: Bram E G, ed. Infrared and Raman Spectroscopy, Part C, New York and Basel: Marcel Dekker Inc. 1977. 717 872

7 Erfurth Stephen C, Peticolas Warner L. M elting and premelting phenomenon in DNA by laser raman scattering. Biopolymers, 1975, 14: 247 264

8 Tu Anthony T. In Raman Spectroscopy in Biology Principles and Applications. New York: John Wiley and Sons Inc, 1982. 165

9 Halliw ell Barry, Aruoma Okezie I. DNA and Free Radicals. England: Ellis Horw ood Limited, 1993. 41 65

10 Zhang $\mathrm{Z}$, Wang $\mathrm{N} \mathrm{H}$, Wan Q, et al. EPR studies of oxygen $\left({ }^{1} \mathrm{O}_{2}\right)$ and free radicals $\left(\mathrm{O}_{2}^{-},{ }^{-} \mathrm{OH} \mathrm{HB}^{--}\right)$generated during photosensitization of hypocrellin B. Free Radical Biology\& Medicine, 1993, 14: $1 \sim 9$

11 Zou W, An J Y, Jiang L J. Damage to pBR322 DNA photosensitized by hypocrellin A in liposomes and its derivative in solution. J Photochem and Photobio, B: Biology, 1996, 33: 73 78

12 M ikinori Kuw abara, Zhang, Z Y, Giichi Yoshii. E S R of spirt trapped radicals in aqueous solutions of pyrimidine nucleosides and nucleotides, reactions of the hydroxyl radical. Int J Radiat Biol, 1982, 41(3) : 241 259

\title{
阿藿烯(Z_Ajoene) 诱导肿瘤细胞凋亡
}

$$
\begin{aligned}
& \text { 张四清赵嵩闵吉梅 (4) 王灌 }{ }^{(4)} \\
& \text { 刘士廉 郑德先 }
\end{aligned}
$$

( 中国医学科学院中国协和医科大学医学分子生物学国家重点实验室, 北京 100005; (4北京医科大学药学院 天然药物与仿生药物国家重点实验室, 北京 100083; (仙中国医学科学院中国协和医科大学实验血液学国家重点实验室,

天津 $300020 . *$ 联系人)

摘要为了研究大蒜的药理作用, 从大蒜中分离得到了含硫的简单有机化合物阿雚烯 (z_Ajoene). 在体外以 不同浓度的阿雚烯处理 3 种不同的肿瘤细胞, 应用 MTT 法分析阿乼烯对肿瘤细胞生长的影响, 采用光学显 微镜、流式细胞仪和琼脂糖凝胶电泳等技术分析肿瘤细胞形态、DNA 含量和染色体 DNA 的断裂, 使用 Western 蛋白印迹法测定基因表达. 发现阿雚烯对 HL_60, M Gc_803 和 Molt_4 等 3 种肿瘤细胞均具有明显的致调 亡作用, 其机制可能是通过抑制原癌基因 bcl_2 的表达而实现的.

\section{关键词 肿瘤细胞凋亡 阿蕉烯 bcl_2 表达}

长期以来, 人们认识到大蒜及其提取物具有抗癌 ${ }^{[1]}$ 、抗细菌、抗真菌感染 ${ }^{[2]}$ 以及抗血栓形 成的作用 ${ }^{[3]}$, 但其药理作用机制及有效成分则不清楚. 本研究从大蒜提取物中分离纯化了阿 藿烯(Z_A joene), 它是一种含硫简单有机化合物, 结构式为 $\mathrm{CH}_{2}=\mathrm{CH}-\mathrm{CH}_{2}-\mathrm{S}(\mathrm{O})-\mathrm{CH}_{2}-$ $\mathrm{CH}=\mathrm{CH}-\mathrm{S}-\mathrm{S}-\mathrm{CH}_{2}-\mathrm{CH}=\mathrm{CH}_{2}$, 分子量为 234. 本项体外实验研究首次证明阿藿烯可诱 导肿瘤细胞凋亡, 并探讨了其作用机制. 


\section{1 材料与方法}

人早幼粒白血病细胞系 HL_60、人 T 淋巴母细胞系 Molt_4 培养于含 $10 \%$ 胎牛血清的 RPM I 1640 培养液; 人胃癌细胞系 M Gc_803, NIH_3T 3 正常成纤维细胞系培养于含 $10 \%$ 胎牛 血清的 DMEM 培养液中. 培养条件为 $5 \% \mathrm{CO}_{2}, 37^{\circ} \mathrm{C}$.

以 MTT 法分析阿蒦烯对肿瘤细胞生长的抑制作用. 在 96 孔培养板中, 每孔分别接种 $3 \times 10^{3}$ 个 M Gc_803 胃癌细胞、1 $0^{4}$ 个 HL_60 白血病细胞及 $5 \times 10^{3}$ 个 N IH 3T 3 正常细胞, 同时 加入不同浓度的阿蕉烯( $10 \mathrm{mmol} / \mathrm{L}$ 拒存液, 溶于 $100 \%$ 乙醇), 每个浓度平行做 3 孔, 以未处 理的细胞作对照, 培养 $48 \mathrm{~h}$; 加入 $20 \mu_{\mathrm{L}}$ MTS/ PMS 溶液(Promega, MTS 终浓度为 $333 \mu_{\mathrm{g}} /$

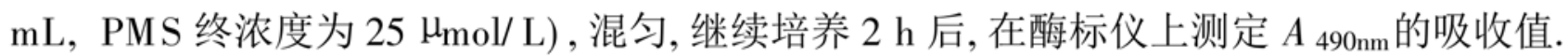

细胞调亡的测定采用如下方法进行. 在光学显微镜下直接观察细胞形态, 或以 $400 \mu \mathrm{L}$ 裂 解液( $1 \%$ NP_40, $20 \mathrm{mmol}$ EDT A, $50 \mathrm{mmol}$ Tris_HCl, $\mathrm{pH}=7.5$ ) 裂解 $10^{6}$ 个细胞, $8000 \mathrm{r} / \mathrm{min}$ 离心 $5 \mathrm{~min}$, 在上清中加入 $10 \% \mathrm{SDS}$ 至终浓度 $1 \%$, 加入 RNase A 至终浓度 $50 \mathrm{\mu g} / \mathrm{mL}, 56{ }^{\circ} \mathrm{C}$ 处 理 $2 \mathrm{~h}$; 加入蛋白酶 $\mathrm{K}$ 至终浓度 $25 \mathrm{\mu g} / \mathrm{mL}$, 消化 $2 \mathrm{~h}$; DNA 于 $1 \%$ 琼脂糖凝胶中电泳, 观察染色 体 DNA 断裂情况. 收获 $10^{6}$ 个细胞, 于 $70 \%$ 乙醇中和 $4{ }^{\circ} \mathrm{C}$ 固定过夜; 离心去上清, 细胞重悬于 $0.4 \mathrm{~mL}$ PBS 中, 加入 $10 \mu_{\mathrm{L}} \mathrm{RN}$ ase $\mathrm{A}(5 \mathrm{mg} / \mathrm{mL}), 37^{\circ} \mathrm{C}$ 消化 $1 \mathrm{~h}$; 然后加 $50 \mu_{\mathrm{L}}$ 的碘化丙啶溶液 $(\mathrm{PI}, 1 \mathrm{mg} / \mathrm{mL}), 4{ }^{\circ} \mathrm{C}$ 染色过夜; 在 FACS420 型流式细胞仪上分析 DNA 含量. 以 Western蛋 白印迹法测定基因表达, 即以细胞裂解液 $50 \mathrm{mmol}$ Tris_HCl, $\mathrm{pH}=6.8,100 \mathrm{mmol}$ DTT, $2 \%$ SDS, $0.1 \%$ 澳酚蓝, $10 \%$ 甘油) 裂解 $5 \times 10^{6}$ 个细胞, $100{ }^{\circ} \mathrm{C}$ 水浴中 $5 \mathrm{~min}$; 每个加样孔中加入 50 $\mu_{\mathrm{g}}$ 蛋白, 经 $12 \%$ SDS_PAGE 分离后, 采用 $30 \mathrm{~V}$ 电压转印 $6 \mathrm{~h} ; \mathrm{NC}$ 膜于 $5 \%$ 脱脂牛奶中封闭 后, 与鼠抗人 Bcl_2 或 P53 单抗 (1:1000 稀释) 孵育过夜. 洗膜后, 再与碱性磷酸酶标记的二抗 ( $1: 2000$ 稀释) 孵育, 然后按照常规以 BCIP/ NBT 显色.

\section{2 实验结果}

2.1 阿蕉烯对肿瘤细胞增殖的抑制作用

如图 1 所示, 阿蕉烯处理细胞 $48 \mathrm{~h}$ 后, 可 显著抑制 HL_60 和 M Gc_803 肿瘤细胞的增 殖. HL_60 白血病细胞、M Gc_803 胃癌细胞和 NIH 3T 3 正常细胞的 $\mathrm{IC}_{50}$ 值分别为 4,36 和 65 $\mu_{\mathrm{mol}} / \mathrm{L}$, 表明肿瘤细胞对阿蒦烯的处理比较敏 感, 而正常细胞不敏感.

\section{2 阿蕉烯诱导肿瘤细胞凋亡}

在光学显微镜下观察的细胞形态如图 2 所示, 未经阿蕉烯处理时, HL_60 细胞 (图 2 (a) ) 为悬浮生长的细胞, 呈圆形, 形态规则饱 满; Molt_4 细胞 (图 2(b) ) 为半贴壁生长的细 胞, 多数呈圆形, 形态规则饱满, 少数为菱形、 棱形; M Gc_803 细胞 (图 2(c)) 为贴壁生长的细 胞, 呈典型的上皮样扁平状. 经阿蕉烯处理

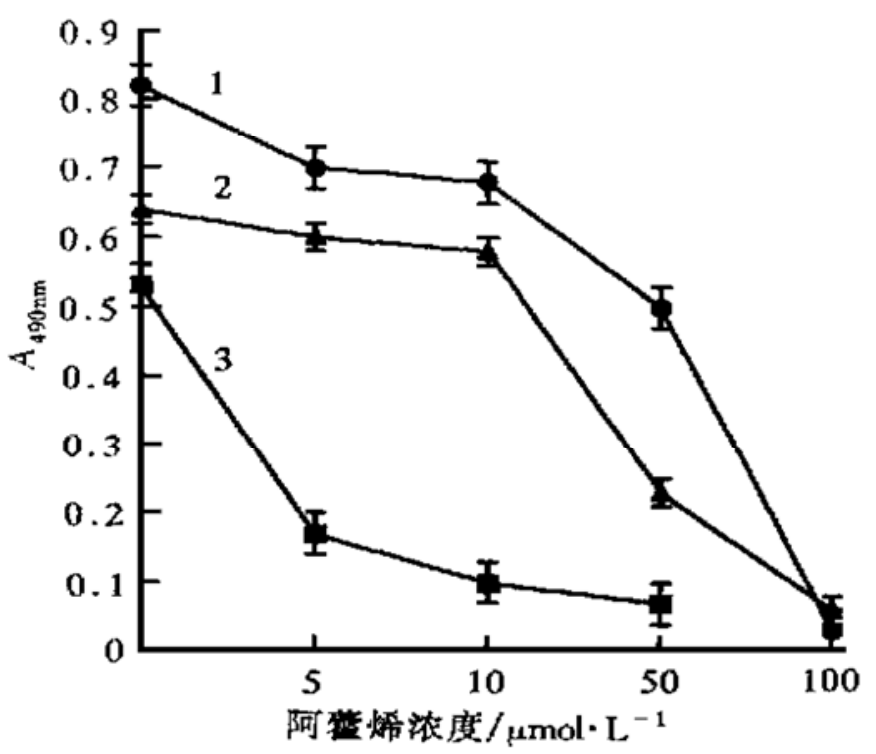

图 1 阿嚄烯对肿瘤细胞和正常细胞生长的抑制作用 1 - NIH3T3 正常细胞; $2-\mathrm{M} \mathrm{Gc}$-803 胃癌细胞; $3-$ HL_60 白血病细胞 
后, HL_60 细胞(图 2(d)) 和 Molt_4 细胞(图 2(e) ) 出现发泡、凋亡小体、皱缩; M Gc_803 细胞 (图 2(f) ) 首先变圆, 然后漂起、发泡, 最后形成调亡小体, 表明阿藿烯可使所试肿瘤细胞发生 明显的细胞调亡现象.

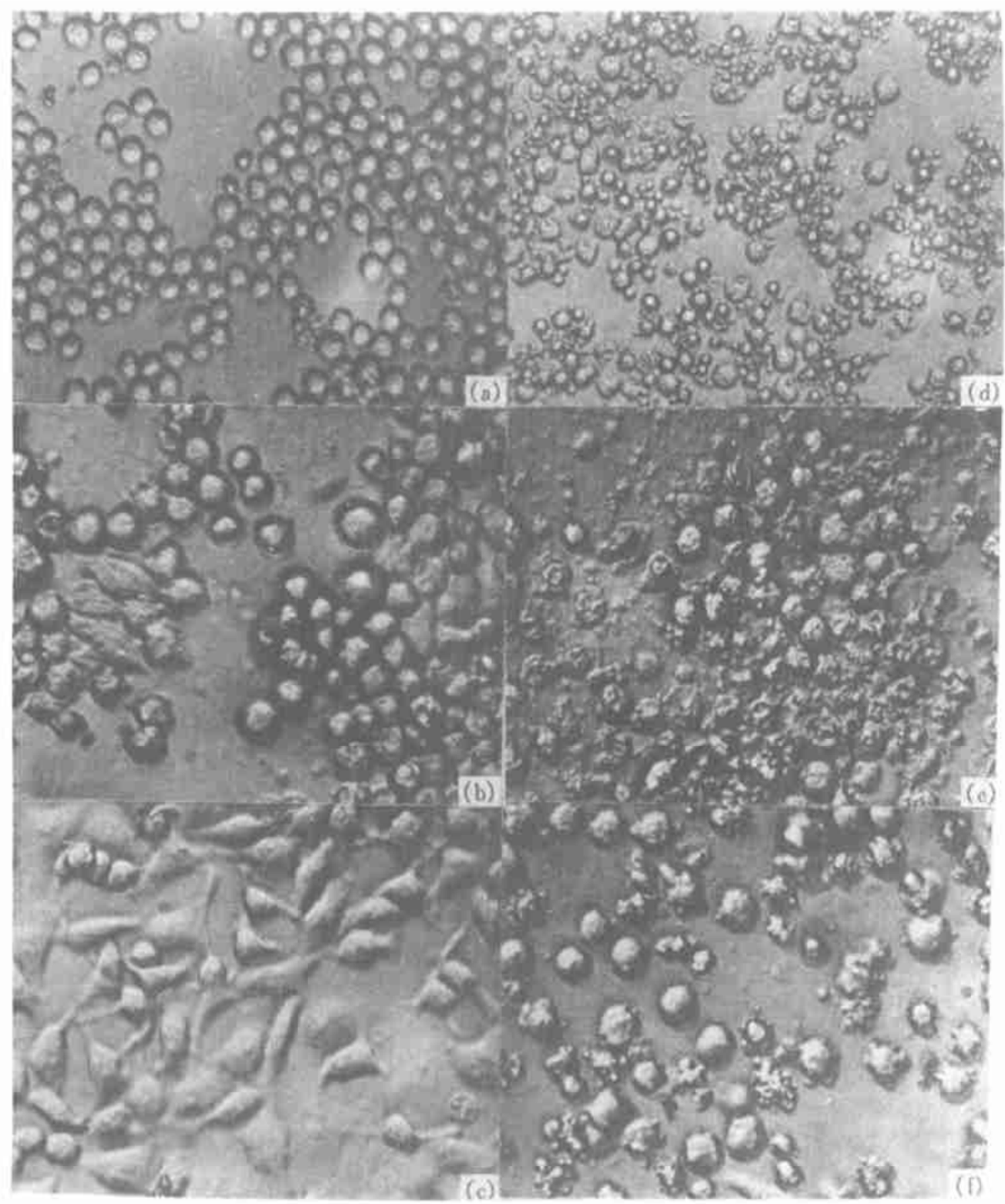

图 2 在光学显微镜下观察的调亡细胞形态 $(\times 200)$

1 HL_60 白血病细胞, $2-\mathrm{Molt}$-4 白血病细胞, 3- $\mathrm{M} \mathrm{Gc}-803$ 胃癌细胞, 4 —经 $10 \mu_{\mathrm{mol} / \mathrm{L}}$ 阿蕉烯处理 $8 \mathrm{~h}$ 的 HL_60 白血病细胞, 5 ——经 $10 \mu \mathrm{mol} / \mathrm{L}$ 阿蒦烯处理 $24 \mathrm{~h}$ 的 Molt_4 白血病细胞, 6 —经 $100 \mu \mathrm{mol} / \mathrm{L}$ 阿蒦烯处理 $15 \mathrm{~h}$ 的 M Gc_803 胃癌细胞

流式细胞仪的分析表明, 以阿蕉烯 $(10 \mu \mathrm{mol} / \mathrm{L})$ 处理 $8 \mathrm{~h}$, 即可诱导 HL_60 白血病细胞出 现典型的 DNA 含量小于 2 倍体的亚 $\mathrm{G}_{1}$ 调亡峰, 同样浓度的阿嚄烯处理 Molt_4 细胞 $24 \mathrm{~h}$ 或 $100 \mu_{\mathrm{mol}} / \mathrm{L}$ 阿蕉烯处理 M Gc_803 胃癌细胞 $15 \mathrm{~h}$, 也可诱导这两种细胞出现调亡(结果未显 
示).

琼脂糖凝胶电泳分析表明, 在上述条件下, 可使 HL_60 白血病细胞 (图 3 孔 4), Molt_4 细 胞(图 3 孔 5) 和 M Gc_803 胃癌细胞(图 3 孔 6) 出现清晰的 DNA 梯状带, 而未处理的对照组细 胞未产生 DNA 梯状带(图 3 孔 1,2,3).

\section{3 阿藿烯对原癌基因 bcl_2 表达的影响}

以 Western 蛋白印迹法分析基因表达的结果如图 4 所示. 50( 图 4 孔 1). 100(图 4 孔 2) 和 $150 \mu_{\mathrm{mol}} / \mathrm{L}$ (图 4 孔 4) 阿藿烯分别处理 M Gc_803 胃癌细胞 $15 \mathrm{~h}$ (其他细胞未做), 与未处理的 细胞(图 4 孔 3) 比较, 发现阿蕉烯的处理使 M Gc_803 胃癌细胞的原癌基因 bcl_2 的表达水平明 显下降, 但不影响 p53 基因的表达(结果未显示), 提示阿藿烯诱导肿瘤细胞凋亡的机制与抑制 Bcl_2 蛋白的表达有关, 而不依赖 p53 基因表达的途径.

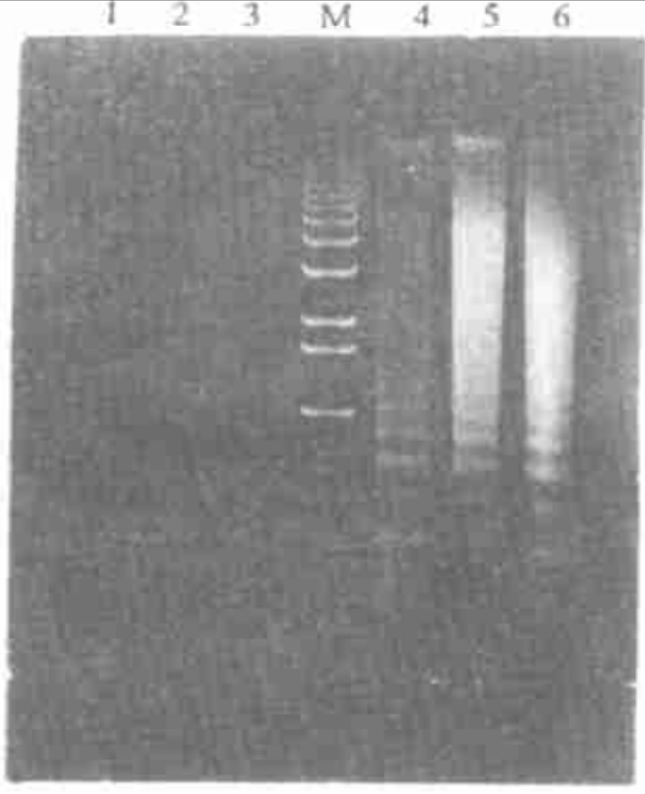

图 3 调亡细胞 DNA 梯状带凝胶电泳结果 $\mathrm{M}$-DNA 分子量标准( $1 \mathrm{~kb}$ DNA ladder). 1 6 同图 2

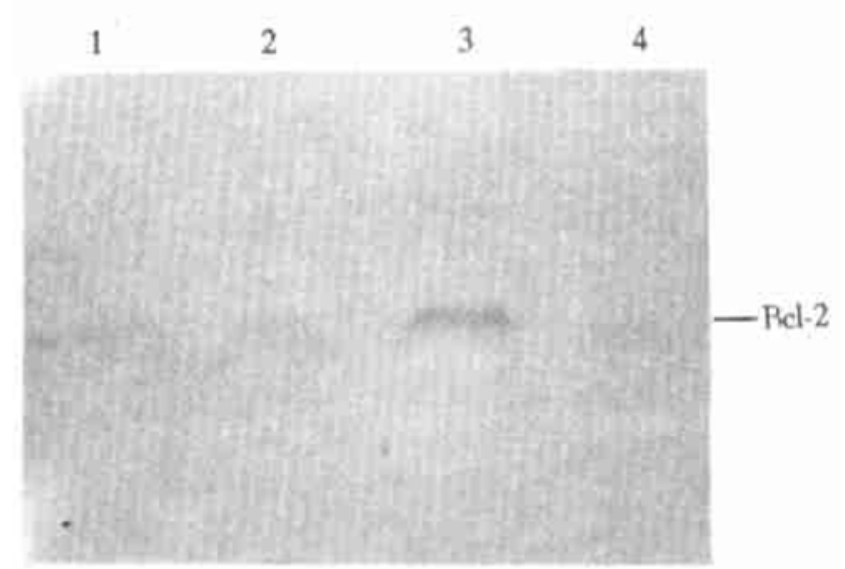

图 4 Western 蛋白印迹显示阿蒦烯抑制 MGc_803 胃癌细胞 Bcl_2 蛋白的表达结果 $1-50 \mu_{\mathrm{mol} / \mathrm{L}}$ 处理 $15 \mathrm{~h}, 2-100 \mu_{\mathrm{mol} / \mathrm{L}}$ 处理 $15 \mathrm{~h}, 3$ 一未处理, $4-150 \mu_{\mathrm{mol}} \mathrm{L}$ 处理 $15 \mathrm{~h}$

\section{3 讨论}

本研究首次发现大蒜中的含硫简单有机化合物阿藿烯可诱导所试 3 种不同肿瘤细胞凋 亡, 这一重要发现表明, 阿嚄烯可能成为一个新的广谱抗肿瘤药物. 进一步采用 Western 蛋白 印迹法研究与细胞调亡密切相关的基因表达, 发现阿蒦烯可抑制肿瘤细胞中原癌基因 bcl_2 的 表达, 但不影响肿瘤抑制基因 p53 的表达. 在约 $80 \%$ 以上的肿瘤细胞中,p53 基因发生了突 变 ${ }^{[4]}$, 使这些肿瘤细胞对传统化疗药物不敏感. 上述结果提示阿藿烯作为非常有希望的新的 化疗药物, 对 p53 基因突变的肿瘤可能更为有效.

原癌基因 bcl_2 又称为抗细胞调亡基因, 可抑制许多因素引起的细胞调亡 ${ }^{[5]}$. 目前, 根据 其 cDNA 序列上的同源性, 已在病毒、线虫和哺乳动物中发现了 bcl 2 的基因家族. 有趣的是 bcl_2 家族的某些基因, 如 bax, bad 和 bcl_ $\mathrm{x}_{\mathrm{s}}$ 等, 却可促进细胞调亡 ${ }^{[6]}$, 这可能与 bcl 2 和 bax 在 
体内的表达水平有关. 因此, 在阿蕉烯诱导的肿瘤细胞调亡过程中, 进一步研究 bcl_2 和 bax 表达的相关性, 将有助于深入了解肿瘤细胞调亡的分子机制.

致谢本工作为国家自然科学基金( 批准号: 39470160)、卫生部基金(合同号: 94_1_017) 和中国医学科学院基 金( 批准号: 931008) 资助项目。

\section{参 考 文 献}

1 Belman S. Onion and garlic oils inhibit tumor promotion. Carcinogenesis, 1983, 4: $1063 \sim 1067$

2 Feldberg R S, Chang S C, Kotik A N, et al. In vitro mechanism of inhibition of bacterial cell grow th by allicin. Antimicrobiol Agents Chemother, 1988, 32(12): $1763 \sim 1768$

3 Apitz Castro R, Badimon J J, Badimon L. A garlic derivative, ajoene, inhibits platelet deposition on severely damaged vessel $\mathrm{w}$ all in an in vivo porcine experimental model. Thromb Res, 1994, 75(3): 243 249

4 Hartwell L H, Kastan M B. Cell cycle control and cancer. Science, 1994, 266: $1821 \sim 1825$

5 Sentman C L, Shutler J R, Hockenberg O, et al. Bcl_2 inhibits multiple forms of apoptosis but not negative selection in thymocytes. Cell, 1991, 67: 879 888

6 Martin S J, Green D R. Apoptosis and cancer: the failure of controls on cell death and cell survival. Critical Rev in Oncol/ Hematol, 1995, 18: 137 153

\section{运用差异显示法分离冬小麦春化 作用相关 cDNA 克隆}

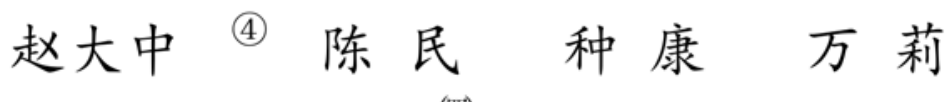
黄华梁 ${ }^{(14)}$ 谭克辉

( 中国科学院植物研究所, 北京 100093; (4北京大学生命科学学院细胞生物学与遗传学系, 北京 100871;

(西)中国科学院遗传研究所, 北京 100101)

摘要 在冬小麦的春化进程中存在着一个核酸与蛋白质代谢的关键期. 为了探讨春化作用的分子机理, 采用 mRNA 差异显示技术, 对来源于冬小麦燕大 1817 不同春化处理的胚芽内的 mRNA 变化进行了检测, 发现了 一个仅在春化 20d 这一关键期表达, 而在未春化、春化 4d、脱春化期不表达的春化相关 cDNA 克隆 VRC54. Northern 杂交及同源性分析进一步表明 VRC54 是一个在植物中新发现的春化特异克隆, 它不仅对春化需求 型植物的成花诱导可能起重要作用,而且不同于冷诱导基因.

\section{关键词 mRNA 差异显示 春化相关 cDNA 克隆 冬小麦}

春化作用是低温需求型植物成花所必需的发育阶段, 尽管人们对春化作用的机理已经做 了大量的研究探讨, 但目前还不清楚其调控植物成花的分子机制. 遗传学研究表明, 春化作用 特性至少由 4 个基因控制 ${ }^{[1]}$. 冬小麦在春化进程中, 虽然发生了一系列的生理生化变化 ${ }^{[2,3]}$, 但存在着一个核酸、蛋白质合成的关键期 ${ }^{[2,4]}$. 进一步的研究发现, 在冬小麦春化关键期新合 成的 mRNA、蛋白质与成花潜力的获得相关 ${ }^{[5,6]}$, 这说明春化作用是一个极其复杂的多基因 控制的过程. 尽管我们实验室已通过差异笁选技术克隆了 2 个春化相关基因 verc17 与 